\title{
ESTUDO DE LAMINADOS DE MATERIAIS COMPÓSITOS NO TRANSPORTE AÉREO
}

\author{
K. C. Dubiella ${ }^{1}$; D. F. Vicentini ${ }^{2}$ \\ ${ }^{1,2}$ Universidade Federal do Paraná, Av. Coronel Francisco H. dos Santos, 210, \\ Jd. das Américas, Curitiba/PR, CEP 81530-000 \\ kauanedubiella@ufpr.br',vicentini@ufpr.br ${ }^{2}$
}

\begin{abstract}
Resumo: Com a crescente demanda do transporte aéreo, esforços têm sido feitos em busca de tecnologias que aumentem a viabilidade e uso deste modal. Nesse sentido, a indústria da aviaçáo tem investido em estruturas laminadas de materiais compósitos em aeronaves, em vista de que esse tipo de material apresenta elevada rigidez e resistência mecânica frente a esforços que submetem a estrutura à compressão e à tração, aliados ao seu baixo peso específico, se comparado com os tradicionais materiais metálicos. Esse comportamento é decorrente da união entre as fibras e a matriz, onde a matriz é a responsável pela proteção da fibra, garantindo boa resistência à corrosáo, à fadiga e maior tenacidade, além de apresentar uma fase contínua responsável por distribuir uniformemente a carga aplicada na estrutura. No presente trabalho, foram estudadas diversas configuraçôes de laminados de fibra de carbono, sujeitos às solicitaçôes normais de tração e compressão, a fim de que se pudesse obter o melhor resultado no dimensionamento de estruturas laminadas. Para a análise, foi implementado um algoritmo em linguagem Fortran, utilizando análise matricial no âmbito da Teoria de Laminados e considerando diversos critérios de falha. Após verificaçôes com o modelo, o algoritmo implementado foi aplicado a uma estrutura aeronáutica real. Ao fim, conclui-se que as orientações que solicitam as fibras longitudinalmente apresentarão o melhor comportamento quando o laminado é submetido a esforços de tração, segundo a maioria dos critérios analisados.
\end{abstract}

Palavras-chave: Compósitos, Laminados, Transporte aéreo.

\begin{abstract}
With the growing demand of aerial transportation, several efforts have been carried out in searching technologies which increase the viability and use of this modal. Following this research line, the aeronautical industry has been investing in the study of laminated structures of composite materials for aircrafts. The material offers high stiffness, and mechanical resistance when subjected to normal loads, with the lower properties in its specific weight, when compared with the traditional metallic materials. This behavior results of the union between fibers and matrix. The matrix is responsible for protection of the fiber, ensuring good corrosion and fatigue resistance. In this work, several configurations of carbon fiber laminated subjected to tensile and compression stresses were studied. An algorithm using laminate theory and matrix analysis was implemented in Fortran. The algorithm considered several failure criteria. After some verification with the implemented model, it was applied to a real aeronautical structure. From this work, it can be concluded that the orientations which present the best behavior in failure are the configurations in which the fibers are subjected to tensile stresses.
\end{abstract}

Keywords: Composites, Laminated, Air transport. 


\section{Introduçáo}

O meio de transporte é um fator impactante nas atividades econômicas e na logística das operaçôes, visto que afeta a viabilidade do escoamento de cargas para determinadas regiôes e disponibilidade de serviços, ou tempo médio de entrega e viagens [1]. Basicamente, a escolha do modal a ser utilizado impacta diretamente nos custos e consequentemente na competitividade desse produto ou serviço. Dentre os modais, o transporte aéreo pode se caracterizar como um modal com custo superior, com a necessidade de um alto investimento inicial, de modo que a oferta do mesmo se localizará em regiôes estratégicas, que sejam lucrativas e que possuam demanda adequada. Esse modal possibilita ainda transportes intercontinentais de modo rápido, o que o torna bastante atrativo. Segundo [2], "desde 2010 o avião tem sido o principal meio de transporte utilizado pelos passageiros nas viagens interestaduais, quando considerados os serviços de transporte regular dos modais aéreo e rodoviário". Essa demanda elevada incentiva o desenvolvimento de novas tecnologias, como, por exemplo, o uso de materiais compósitos laminados, já que esse material permite o projeto de estruturas leves com alta capacidade de carga, como por exemplo, a criação da nova série de aeronaves da Airbus [3], possibilitando a reduçáo do custo desse serviço.

Os compósitos são formados pela união de dois materiais que constituirão um material com propriedades não alcançadas pelos mesmos separadamente. A utilização dos materiais compostos modernos teve amplo emprego na área aeronáutica a partir de 1960 [4], conferindo às estruturas uma redução no peso dos componentes estruturais e resistência comparável ou até mesmo superior a alguns metais utilizados na aviação [5]. A leveza das estruturas aeronáuticas também acarreta em uma menor geração de poluentes pelo fato de demandar uma menor quantidade de combustíveis e permitir o transporte de maior quantidade de passageiros e/ou carga por viagem, trazendo assim mais eficiência ao modal.

Basicamente, o trabalho em questão visa estudar determinadas configurações geométricas de estruturas de laminados a fim de que se garanta um comportamento ótimo da estrutura. Pretende-se, então, através da aplicação de um algoritmo, analisar quais são as geometrias que apresentam o melhor comportamento, sob um mesmo nível de solicitação. A análise está baseada nas respostas de aviso de falha segundo os principais critérios de resistência conhecidos na literatura sobre materiais compósitos, fundamentada na Teoria de Laminados.

\subsection{Materiais compósitos}

Os materiais compostos possuem um sistema de eixos de ortotropia, como pode ser observado na Fig. 1, sendo a direção 1 paralela às fibras, 2 a direção perpendicular às fibras e 3 a direção perpendicular às direçóes 1 e 2 simultaneamente. Os índices das constantes elásticas são denominados a partir desse sistema de eixos [6]. Para a análise de um laminado, primeiramente um eixo de referência $(\mathrm{x}, \mathrm{y}, \mathrm{z})$ é definido, a fim de referenciar o posicionamento das lâminas. Basicamente, ao analisar o 
laminado, a matriz constitutiva do material deve ser calculada segundo os eixos de ortotropia e de referência, os termos de acoplamento, coeficientes de temperatura no eixo de referência, deformaçôes, tensôes e constantes elásticas. Após, os critérios de ruptura ou métodos de degradação podem ser aplicados.

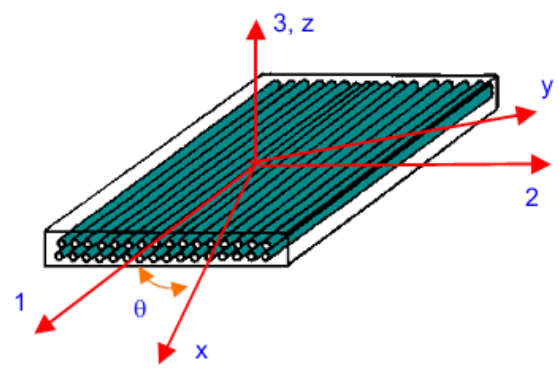

Figura 1 - Sistema de eixos de ortotropia e de referência.

Fonte: [4].

\subsection{Critérios de ruptura}

A resistência do laminado é determinada pelo comportamento das lâminas em conjunto, ou seja, resistência e rigidez das lâminas que o compóe, coeficiente de dilatação, orientação, espessura, sequência de empilhamento e temperatura de cura. A direção de aplicação da carga também estabelece quais serão as lâminas que irão resistir, de modo que quando uma lâmina falha em serviço, outras lâminas poderão resistir, fazendo com que a estrutura não se rompa completamente. Os mecanismos de ruptura do material composto podem ter diferentes motivos: ruptura das fibras, ruptura da matriz, separação da fibra/matriz, separação das lâminas, entre outros [4]. A seguir são apresentados brevemente alguns dos principais critérios de ruptura. Todos os critérios garantem que a lâmina, ao ser submetida a tal estado de tensão não se romperá [4]. Um dos critérios mais conhecidos é o da Máxima tensão, definido de acordo com as seguintes relaçôes:

$$
X_{c}<\sigma_{1}<X_{t} \quad, \quad Y_{c}<\sigma_{2}<Y_{t} \quad \text { e }^{-S<\tau_{12}<S}
$$

onde $X_{t}$ e $X_{c}$ são as resistências longitudinais à tração e à compressão, $Y_{t}$ e $Y_{c}$ representam as resistências transversais à tração e à compressão e $S$ é a resistência ao cisalhamento. $O$ critério da Máxima Deformação estabelece que:

$$
\varepsilon_{1}<X_{\varepsilon t} \text { ou }\left|\varepsilon_{1}\right|<X_{\varepsilon c}, \varepsilon_{2}<Y_{\varepsilon t} \text { ou }\left|\varepsilon_{2}\right|<Y_{\varepsilon c} \text { e } \gamma_{12}<S_{\varepsilon}
$$


em que $X_{\llbracket c}$ e $X_{\square t}$ representam as deformaçóes máximas na direçáo longitudinal em compressão e tração; $Y_{\varpi c}$ e $Y_{\varpi t}$ representam deformações máximas na direção transversal em compressão e tração e $S_{\square}$ se refere à deformação máxima em cisalhamento. $\mathrm{O}$ critério de Hill é obtido ao igualar a energia de distorção de cisalhamento com a energia no ponto de ruptura [4], representado pela Eq. (3):

$$
\left(\frac{\sigma_{1}}{X}\right)^{2}+\left(\frac{\sigma_{2}}{Y}\right)^{2}-\left(\frac{1}{X^{2}}+\frac{1}{Y^{2}}-\frac{1}{Z^{2}}\right) \sigma_{1} \sigma_{2}+\left(\frac{\tau_{12}}{S_{12}}\right)^{2}=1
$$

O critério de Tsai-Hill, apenas iguala os eixos y e z, simplificando a expressão do critério de Hill [4], definindo assim a seguinte condição:

$$
\left(\frac{\sigma_{1}}{X}\right)^{2}+\left(\frac{\sigma_{2}}{Y}\right)^{2}-\left(\frac{\sigma_{1} \sigma_{2}}{X^{2}}\right)+\left(\frac{\tau_{12}}{S_{12}}\right)^{2}=1
$$

O critério de Hofman admite que a ruptura acontece quando a seguinte igualdade é verificada [6]:

$$
\frac{\sigma_{1}^{2}}{X_{t} X_{c}}+\frac{\sigma_{2}^{2}}{Y_{t} Y_{c}}-\frac{\sigma_{1} \sigma_{2}}{X_{t} X_{c}}+\frac{X_{c}-X_{t}}{X_{t} X_{c}} \sigma_{1}+\frac{Y_{c}-Y_{t}}{Y_{t} Y_{c}} \sigma_{2}+\left(\frac{\tau_{12}}{S_{12}}\right)^{2}=1
$$

Para Hashin e Rotem, a ocorrência da falha de um compósito depende dos mecanismos de falha do mesmo [6]. Os critérios de Hashin-Rotem [6] consideram um estado biaxial de tensôes, onde é possível perceber que as sentenças são diferenciadas para cada modo de falha. $\mathrm{O}$ critério foi formulado em termos de equaçóes, expressas na Tabela 1.

Tabela 1 - Critérios de Hashin-Rotem.

\begin{tabular}{cc}
\hline Modo de falha & Hashin-Rotem (73) \\
\hline Falha da fibra em tração (FFT) & $\sigma_{11}=X_{t} \mid \sigma_{11}, X_{t}>0$ \\
Falha da fibra em compressão (FFC) & $\left|\sigma_{11}\right|=X_{c} \mid \sigma_{11}<0$ e $X_{c}>0$ \\
Falha da matriz em tração (FMT) & $\left(\frac{\sigma_{12}}{S}\right)^{2}+\left(\frac{\sigma_{22}}{Y_{t}}\right)^{2}=1 \mid \sigma_{22}>0$ \\
Falha da matriz em compressão & $\left(\frac{\sigma_{12}}{S}\right)^{2}+\left(\frac{\sigma_{22}}{Y_{c}}\right)^{2}=1 \mid \sigma_{22}<0$ \\
\hline
\end{tabular}




\section{Metodologia}

Para a análise de ruptura de laminados, no presente trabalho foi desenvolvido um algoritmo em ambiente Microsoft Visual Studio, cuja compilação foi possível graças à sua integração com o Intel Fortran Compiler. Ao término da programação, foi gerado um aplicativo que permite fazer a utilização do algoritmo sem a necessidade de um ambiente de programação e compilador instalados. Ao executá-lo, é necessária a existência de um arquivo de entrada que deve ser fornecido pelo usuário seguindo recomendaçôes encontradas no manual de utilização do algoritmo. A partir da leitura dos dados contidos no arquivo de entrada, todos os cálculos necessários são efetuados, gerando, ao final do processo, os arquivos com os resultados, segundo todos os critérios implementados (Máxima Tensão, Máxima Deformação, Hill, Hashin-Rotem, Tsai-Hill, Hofman). O algoritmo está baseado nos estudos referentes à Teoria de Laminados e análise matricial, e foi calibrado, inicialmente, com exemplos obtidos na literatura [6].

Após serem verificadas as respostas segundo as opçóes propostas, foi feita a análise frente aos critérios aplicados para laminados nas seguintes configuraçôes: $\left[0^{\circ}\right]_{4},\left[0^{\circ}\right]_{8},\left[0^{\circ} / 90^{\circ}\right]_{s},\left[0^{\circ} / 90^{\circ}\right]_{2}$, $\left[+15 \% /-15^{\circ}\right]_{s},\left[+30^{\circ} /-30^{\circ}\right]_{s},\left[+45 \% /-45^{\circ}\right]_{s},\left[+60 \% /-60^{\circ}\right]_{s}$. O uso de diferentes configuraçóes é justificado, uma vez que estruturas laminadas podem apresentar diferentes comportamentos quando solicitados, dependendo da configuração. Assim que a primeira falha em uma lâmina é identificada sob algum critério, a análise avança para uma segunda etapa que consiste em aplicar métodos de degradação nas lâminas. Em seguida todas as propriedades mecânicas são recalculadas considerando as alteraçôes referentes ao passo anterior, e assim, novamente os critérios de ruptura são aplicados. Quando o algoritmo aplica os critérios de ruptura para avaliar se haverá falha na segunda lâmina, apenas são verificados os critérios que acusaram falha na primeira lâmina.

\section{Resultados}

Inicialmente, a estrutura escolhida para análise consistiu em uma estrutura real, ensaiada em laboratório, com as características descritas na Tabela 2 [7]. No algoritmo, foi considerada a aplicação de uma carga de $1000 \mathrm{KN}$ na direção x (conforme sistema de eixos definidos na Fig. 1), em tração. Os resultados obtidos com o algoritmo implementado são apresentados na Tabela 3 e nos gráficos gerados (a) a (d), para alguns dos principais critérios, em função da orientação das lâminas com relação à direção de solicitação (onde tração e compressão são representadas em módulo). 
Tabela 2 - Propriedades Mecânicas do Laminado analisado.

\begin{tabular}{cccc}
\hline \multicolumn{3}{c}{ Propriedades Mecânicas do Laminado } \\
\hline \multicolumn{2}{c}{ Lâmina } & \multicolumn{2}{c}{ Laminado } \\
Módulos de Elasticidade & 141300 & Resistências admissíveis (MPa) \\
$\mathrm{E}_{1}(\mathrm{MPa})$ & 9850 & $\mathrm{Xt}$ & 1800 \\
$\mathrm{E}_{2}(\mathrm{MPa})$ & 9850 & $\mathrm{Xc}$ & 1300 \\
$\mathrm{E}_{3}(\mathrm{MPa})$ & $\mathrm{Yt}$ & 69,07029743 \\
$\mathrm{Módulo} \mathrm{de} \mathrm{Cisalhamento}$ & $\mathrm{Yc}$ & 49,8841037 \\
$\mathrm{G}_{12}(\mathrm{MPa})$ & 5000 & $\mathrm{~S}$ & 109.28571 \\
$\mathrm{Coeficiente}$ de Poisson & & \\
$v_{12}$ & 0.3 & & \\
$v_{21}$ & 0.020913 & &
\end{tabular}

Nos casos dos critérios de Máxima Tensão, Máxima Deformação e Hashin-Rotem (FFT/FFC), as equaçóes consideram apenas as componentes referentes à tensão observadas nas direçóes x e y do eixo de referência separadamente. Compreende-se que tal fato é uma desvantagem do critério, visto que o material não se deforma de modo isotrópico e que, ante a solicitação normal em x ocorrem deformaçôes em outras direçôes. Desse modo, os mesmos podem ser considerados simplistas, pois analisam as respostas em cada direção separadamente e não consideram o acoplamento entre esforços. Outro fato a destacar é que os critérios de Máxima Tensão e Máxima Deformação não retornam o mesmo resultado. Isso ocorre devido ao fato de que, no caso da limitação da deformação, é feita uma conversão (segundo a Lei de Hooke Generalizada) utilizando o coeficiente de Poisson associando os dados obtidos a partir do ensaio de tração, que fornece um valor de resistência mecânica admissível em determinada direção, obtida a partir da carga aplicada e dos deslocamentos medidos. 
Tabela 3 - Resultados com aplicação de carga N=1000KN em tração em x. Gráficos (a) a (d) mostram as tensóes que provocaram a falha (tração, e compressão em módulo) para alguns critérios, e diversas orientaçôes de laminados.

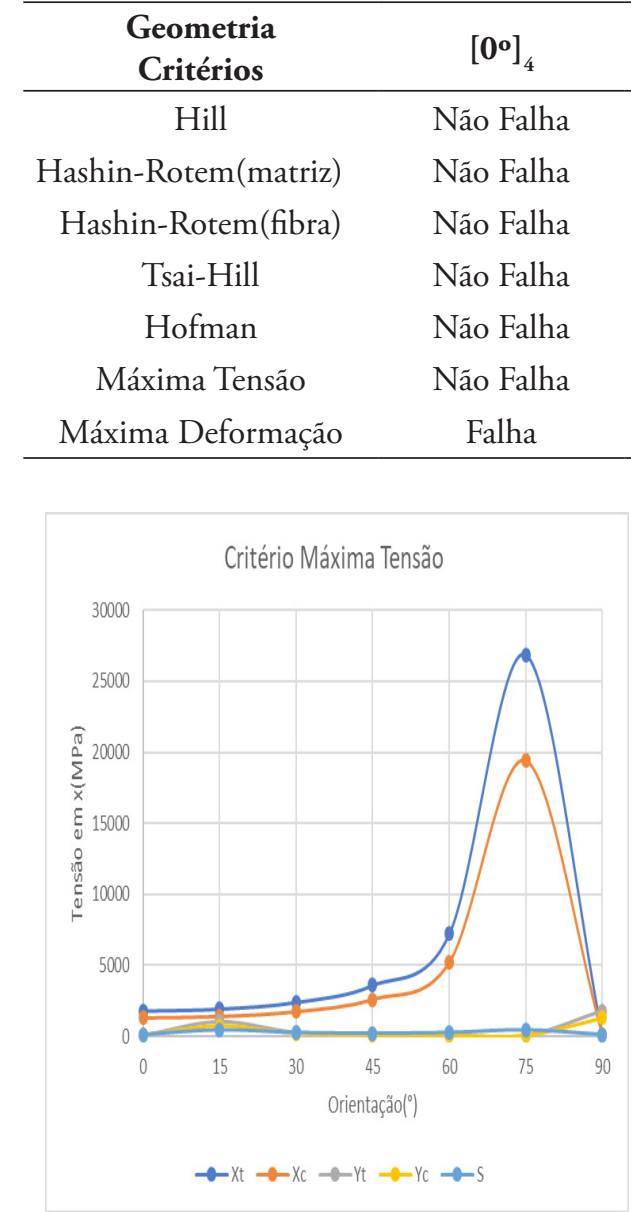

(a)

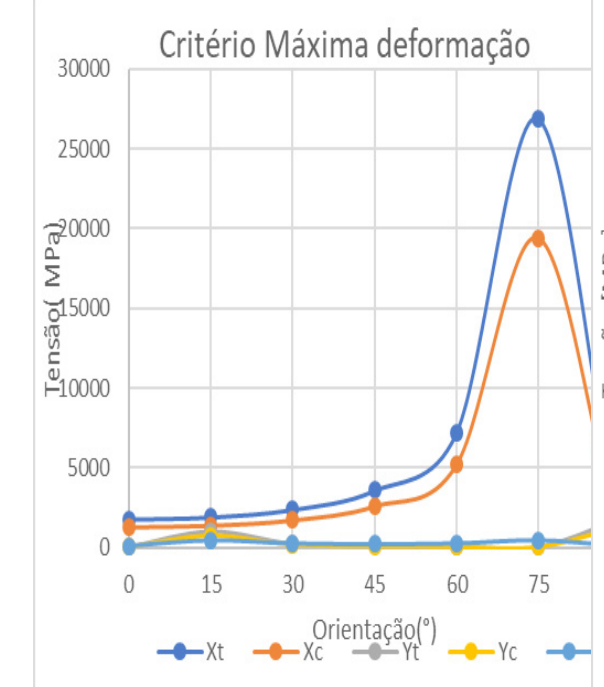

(b)

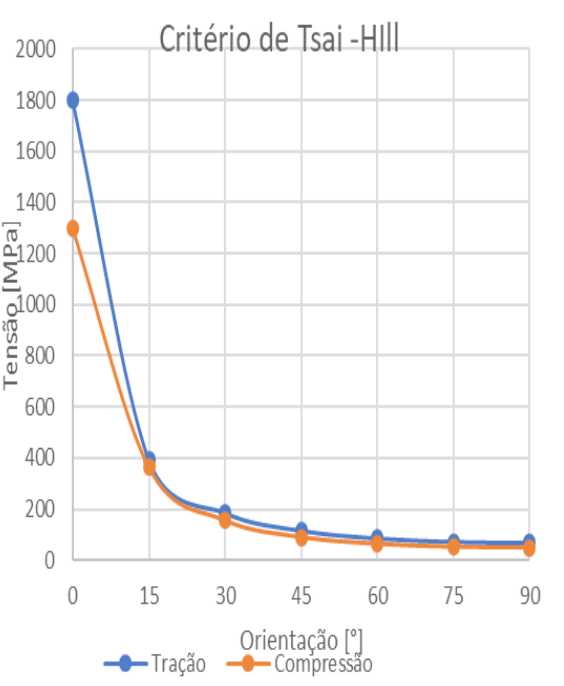

(c)

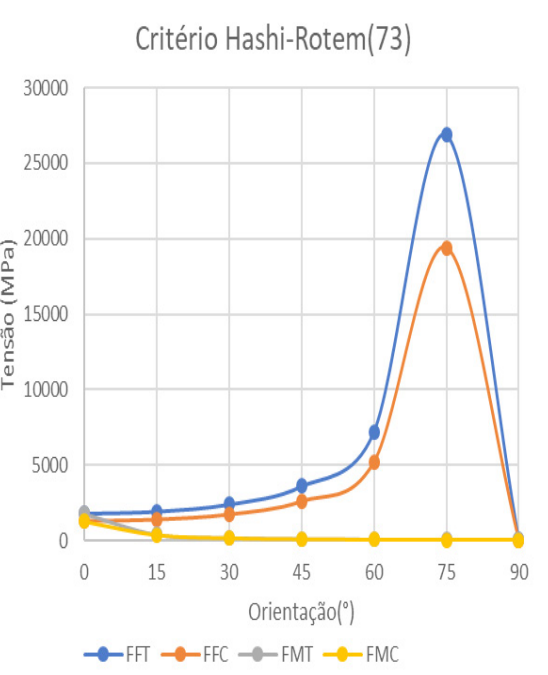

(d) 
Entretanto, o critério de Máxima Tensão acusa falha apenas nas direçóes nas geometrias $\left[+45^{\circ} /-45^{\circ}\right]_{s,}\left[+60 \% /-60^{\circ}\right]_{s .}$ Essa resposta é compreensível, visto que as fibras são as responsáveis pela resistência mecânica da lâmina e assim, ao impor a existência de uma inclinação destas com a direção do carregamento, as fibras passam a ser solicitadas em direçóes não principais, ou seja, com valores de resistência inferiores, favorecendo a falha da estrutura. Esse fenômeno é acentuado quanto maior é a inclinação apresentada (configuraçóes $\left[+45^{\circ} /-45^{\circ}\right]_{s,}\left[+60^{\circ} /-60^{\circ}\right]_{s}$ ). Isso é reafirmado nos gráficos a) e b) da Tabela 3, onde observa-se que o laminado possui uma boa resposta nas orientaçóes próximas à de $0^{\circ}$. Vale destacar que, apesar de os gráficos apresentarem um valor de tensão extremamente elevado a $75^{\circ}$ para os valores referentes de resistência na direção X em tração e compressão, o critério considera que a tensão deve ser o menor valor entre todos os apresentados, assim essa descontinuidade pode ser ignorada. O mesmo fenômeno ocorre para o critério de Máxima Deformaçáo e Hashin-Rotem.

Como indicado na Tabela 3, o critério de Máxima Deformação acusa falha em todas as geometrias, sendo o mais conservador dentre os estudados. Ainda de acordo com os resultados (Tabela $3)$, é possível observar que as configuraçôes de laminados unidirecionais $\left(\left[0^{\circ}\right]_{4}\right.$ e $\left.\left[0^{\circ}\right]_{8}\right)$, cruzados $\left(\left[0^{\circ} / 90^{\circ}\right]_{S}\right.$ e $\left.\left[0^{\circ} / 90^{\circ}\right]_{2}\right)$, bem como as configuraçóes simétricas $\left[+15 \% /-15^{\circ}\right]_{S}$ e $\left[+30^{\circ} /-30^{\circ}\right]_{S}$ não falharam após a aplicação da carga de $1000 \mathrm{KN}$, exceto pelo critério da Máxima Deformação, que indicou a falha prematura para todas as configuraçôes avaliadas. Os resultados para os critérios de Hill e Hashin-Rotem mostraram ser os menos conservadores dentre os analisados, indicando a falha na fibra somente para a configuração $\left[+45 \%-45^{\circ}\right]_{S}$. De modo geral, dentre as configuraçôes avaliadas, as simétricas $\left[+45 \% /-45^{\circ}\right]_{S}$ e $\left[+60^{\circ} /-60^{\circ}\right]_{S}$ foram as menos resistentes, devido ao fato de que as fibras são as responsáveis por fornecerem ao laminado a propriedade mecânica de resistir à tração, e nessas geometrias as mesmas são solicitadas em direçôes "não longitudinais" às fibras, que possuem menor capacidade de resistir à tração.

O critério de Hill considera em uma única equação as deformações causadas em outras direçôes devido a um carregamento na direção x. Em suma, esse critério iguala a energia de distorção de cisalhamento com a energia no ponto de ruptura [4]. Ao observar a Tabela 3, esse critério não indicou a falha de nenhuma lâmina. Já o critério de Tsai-Hill, considera as energias de deformação associadas à direçáo 3 (equivalente à direção z) [6]. Os dois critérios, de Tsai-Hill e Hill, consideram a existência de interação entres tensôes tangenciais e normais, além de diferenciarem o comportamento à tração e à compressão. Observando a Tabela 3, o critério de Hill não acusou falha em nenhuma das geometrias. No gráfico referente ao critério de Tsai-Hill (Tabela 3, gráfico c)), observa-se que a melhor configuração é a de $0^{\circ}$, que favorece que as fibras sejam solicitadas apenas na direção longitudinal. Outro ponto a destacar é o fato de que o valor de resistência mecânica à compressão (em módulo) é inferior ao valor da tração, visto que a compressão é um esforço desestabilizador.

O critério de Hofman considera as diferenças entre comportamentos de tração e compressão, sendo baseado no critério de Hill, anteriormente comentado [2]. Para este critério, o algoritmo indicou falha apenas para as configuraçôes simétricas $\left[+45 \%-45^{\circ}\right]_{s,}\left[+60 \%-60^{\circ}\right]_{s}$, visto que nessas 
condiçôes as lâminas são solicitadas em direções com resistências mecânicas inferiores. Os critérios de Hashin-Rotem preveem a ocorrência da falha baseando seus parâmetros nos modos de falha do compósito [5] (daí a diferenciação entre a falha na fibra FFT/FFC e na matriz FMT/FMC, em tração e compressão respectivamente). Assim, segundo este critério, o algoritmo mostrou que o mecanismo de falha ocorre antes nas fibras do que na matriz, para o laminado $\left[+45^{\circ} /-45^{\circ}\right]_{\mathrm{S}}$.

Em 2006, [6] apresentaram uma interessante crítica quanto à simplicidade destes critérios, salientando a necessidade de um que considere conceitos energéticos. No presente trabalho, algumas dificuldades quanto à simplicidade em cada critério na análise da falha também são relatadas. Basicamente, as aplicaçóes dos critérios nas diferentes orientaçóes utilizam a matriz de transformação para associar os valores referentes às direçôes principais da lâmina com as direçôes do eixo do laminado. Os gráficos apresentados na Tabela 3 levam em consideração as equaçóes dos critérios, fazendo com que o gráfico obtido forneça (em módulo) a máxima tensão suportada na direção $\mathrm{x}$, e todos os valores das tensôes são apresentados em módulo.

\section{Conclusóes}

Os materiais compósitos possuem excelente comportamento quando solicitados por esforços normais, possuindo ampla aplicaçáo em projetos da indústria aeronáutica. Para o desenvolvimento de estruturas otimizadas, no entanto, é preciso a realização de estudos em busca de configuraçôes que permitam a maior capacidade de carga. Em vista disso, neste trabalho foram apresentados alguns dos principais critérios de falha para a análise de laminados de CFRP em diferentes configuraçôes.

Destaca-se o fato de que os critérios de ruptura não evidenciam todos os modos de falha que possam vir a ocorrer, em vista dos parâmetros que são obtidos com os ensaios realizados (ensaio de tração e compressão). Desse modo verifica-se a necessidade da aplicação e análise do critério mais adequado ao comportamento do laminado. Assim, destacam-se os seguintes fatos:

- Os critérios de Tsai-Hill e Hill são melhores aplicados em situações onde é preciso considerar as relaçóes entre o comportamento obtido experimentalmente e o comportamento teórico;

- Os critérios de Hashin e Hashin-Rotem valorizam a análise dos diferentes modos de falha (fibra ou matriz);

- Os critérios de Máxima Tensão e Máxima Deformação tendem a acusar a falha da lâmina antecipadamente em relação aos outros critérios sendo, sendo mais conservadores e simplistas. 
No presente trabalho, o critério de Máxima Deformação em particular, foi o que apresentou a menor capacidade de carga, apesar de visualmente ser muito próximo ao resultado obtido com o critério de Máxima Tensão. O critério de Puck não foi considerado devido ao desconhecimento de alguns parâmetros, - em particular, o fator de magnificação - necessário no cálculo segundo o critério. Porém, os demais critérios considerados associam as tensôes nas três direçóes, o que torna os mesmos mais confiáveis com relação ao comportamento real da estrutura. Em todos os critérios, as configuraçôes com melhores resultados são as que possuem maior solicitação na direção longitudinal da fibra, ou seja, as que possuem orientação com valores igual a $0^{\circ}$ ou próximos de $0^{\circ}$, em vista de que essa direção é a que possui maior resistência admissível do material.

\section{Referências}

[1] BALLOU, R. H. Gerenciamento da Cadeia de Suprimentos: planejamento, organização e logística empresarial. 4. ed. São Paulo: Bookman, 2001.

[2] ANUÁRIO DO TRANSPORTE AÉREO 2016 [da] ANAC, Brasília, 2017, Anual. Disponível em: <http://www. anac.gov.br/assuntos/dados-e-estatisticas/mercado-de-transporte-aereo/anuario-do-transporte-aereo/dados-do-anuariodo-transporte-aereo>. Acesso em: 20 out. 2017.

[3] BARROS, Alexandre G. de; WIRASINGHE, S.c. Designing the airport airside for the new large aircraft. Journal Of Air Transport Management. Calgary, p. 121-127. 01 jan. 2012. Disponível em: <https://www.journals.elsevier. com/journal-of-air-transport-management>. Acesso em: 20 out. 2017.

[4] PEREIRA, J. C. Curso de Projeto Estrutural com materiais Compostos. Florianópolis: UFSC, Departamento de Engenharia Mecânica, 2005.127 p. Disponível em: <http://www.grante.ufsc.br/download/MatCompostos/MATCOMPOSTOS-Apostila-J.Carlos.pdf>. Acesso em: 20 out. 2017.

[5] REZENDE, M. C.; BOTELHO, E. C. O uso de compósitos estruturais na indústria aeroespacial. Polímeros, São Carlos, v. 10, n. 2, p. e4-e10, junho 2000. Disponível em: <http://www.scielo.br/scielo.php?script=sci_ arttext\&pid=S010414282000000200003\&lng=en\&nr m=isso >. Acesso em: 1 mar. 2017.

[6] CARBALLO, F. P.; DELGADO, J. C.; VALLEJO, J. C. M. Introduccion al analisis y diseńo con materiales compuestos. Sevilla: Grupo de Elasticidad y Resistencia de Materiales, 2006. 197 p.

[7] VICENTINI, Daniane Franciesca. Study of the stress state and failure stress state and failure in adhesive joints in adhesive joints with composite materials. 2014. 336 f. Tese (Doutorado) - Mechanical Engineering, Grupo de Elastacidad y Resistencia de Materiales, University Of Seville, Seville, Spain, 2012. Chapter 2. 